\title{
Multisymplectic formulation of fluid dynamics using the inverse map
}

\author{
By C. J. Cotter, D. D. Holm and P. E. Hydon \\ Department of Aeronautics, Imperial College London, London, UK;Department of \\ Mathematics, Imperial College London, London, UK; Department of Mathematics, \\ University of Surrey, Guildford, UK
}

We construct multisymplectic formulations of fluid dynamics using the inverse of the Lagrangian path map. This inverse map - the "back-to-labels" map - gives the initial Lagrangian label of the fluid particle that currently occupies each Eulerian position. Explicitly enforcing the condition that the fluid particles carry their labels with the flow in Hamilton's principle leads to our multisymplectic formulation. We use the multisymplectic one-form to obtain conservation laws for energy, momentum and an infinite set of conservation laws arising from the particle-relabelling symmetry and leading to Kelvin's circulation theorem. We discuss how multisymplectic numerical integrators naturally arise in this approach.

Keywords: Multisymplectic systems, partial differential equations, fluid dynamics, conservation laws, potential vorticity

\section{Introduction}

Definition 1.1. A system of partial differential equations (PDEs) is said to be multisymplectic if it is of the form

$$
K_{i j}^{\alpha}(\boldsymbol{z}) z_{, \alpha}^{j}=\frac{\partial H}{\partial z^{i}}
$$

where each of the two-forms

$$
\kappa^{\alpha}=\frac{1}{2} K_{i j}^{\alpha}(\boldsymbol{z}) \mathrm{d} z^{i} \wedge \mathrm{d} z^{j}
$$

is closed. Here $\boldsymbol{z}$ is an ordered set of dependent variables, total differentiation with respect to each independent variable $q^{\alpha}$ is denoted by the subscript $\alpha$ after a comma, and the Einstein summation convention is used.

The closed two-form $\kappa^{\alpha}$ is associated with the independent variable $q^{\alpha}$; it is analogous to the symplectic two-form for a Hamiltonian ordinary differential equation. Hence there is a symplectic structure associated with each independent variable. In the first of a series of papers, Bridges (1997) pioneered the development of multisymplectic systems, showing that the rich geometric structure that is endowed by the symplectic two-forms can be used to understand the interaction and stability of nonlinear waves. For many important PDEs, the multisymplectic formulation has revealed hidden features that are important in stability analysis. In order to preserve at least some of these features in numerical simulations, Bridges \& Reich (2001) introduced multisymplectic integrators, which generalise the symplectic 
methods that have been widely used in numerical Hamiltonian dynamics. Hydon (2005) showed that multisymplectic systems of PDEs may be derived from Hamilton's principle whenever the Lagrangian is affine in the first-order derivatives and contains no higher-order derivatives. This can usually be achieved by introducing auxiliary variables to eliminate the derivatives.

The aim of this paper is to provide a unified approach to producing multisymplectic formulations of fluid dynamics, based on the inverse map. Our approach covers all fluid dynamical equations that are written in Euler-Poincaré form (Holm et al., 1998), i.e. all equations which arise due to the advection of fluid material. First we use the inverse map to form a canonical Euler-Lagrange equation (following the Clebsch representation given in Holm \& Kupershmidt, 1983, which supports all possible initial conditions for vorticity, in particular flows in three dimensions with non-zero helicity). This approach is different to that of Marsden et al., 2001, where the forward map is used. Then the Lagrangian is made affine in the space and time derivatives by using constraints that introduce additional variables. Following Hydon (2005), we obtain a one-form quasi-conservation law which, when it is pulled back to the space and time coordinates, gives conservation laws for momentum and energy. We also obtain a two-form conservation law that represents conservation of symplecticity; when this is pulled back to the spatial coordinates, it leads to a conservation law for vorticity. The multisymplectic version of Noether's Theorem yields an infinite space of conservation laws from the particle-relabelling symmetry for fluid dynamics; these conservation laws imply Kelvin's circulation theorem. The conserved momentum that is canonically conjugate to the back-to-labels map plays a key role in the derivation of the conservation laws. The corresponding velocity is the convective velocity, whose geometric properties are discussed in Holm et al. (1986). The connection between particle-relabelling symmetry and circulation preservation was first addressed in a multisymplectic context in Bridges et al. (2005).

In this paper we show how the above constructions are made in general, illustrating this with examples. We also discuss how multisymplectic integrators can be constructed using these methods. Sections 2 and 3 review the relations among multisymplectic structures, the Clebsch representation and the momentum map associated with particle relabelling. Section 4 shows how to construct a multisymplectic formulation of the Euler-Poincaré equation for the diffeomorphism group (EPDiff), and derives the corresponding conservation laws, including the infinite set of conservation laws that yield Kelvin's circulation theorem. The section then extends this formulation to the Euler-Poincaré equation with advected quantities. This is illustrated by the incompressible Euler equation, showing how the circulation theorem arises in the multisymplectic formulation. Section 5 sketches numerical issues in the multisymplectic framework. Finally, Section 6 summarises and outlines directions for future research.

\section{Review of multisymplectic structures}

This section reviews the formulation of multisymplectic systems and their conservation laws, following Hydon (2005).

A system of partial differential equation (PDEs) is multisymplectic provided that it can be represented as a variational problem with a Lagrangian that is affine

Article submitted to Royal Society 
in the first derivatives of the dependent variables:

$$
L=L_{j}^{\alpha}(\boldsymbol{z}) z_{, \alpha}^{j}-H(\boldsymbol{z}) .
$$

Remark 2.1. For convenience we retrict attention to autonomous systems where $H$ depends on the dependent variables only; this can always be accomplished by introducing new dependent variables as in the Hamiltonian case.

The Euler-Lagrange equations are then

$$
K_{i j}^{\alpha}(\boldsymbol{z}) z_{, \alpha}^{j}=\frac{\partial H}{\partial z^{i}}
$$

where the functions

$$
K_{i j}^{\alpha}(\boldsymbol{z})=\frac{\partial L_{j}^{\alpha}}{\partial z^{i}}-\frac{\partial L_{i}^{\alpha}}{\partial z^{j}}
$$

are coefficients of the multisymplectic structure matrix. We define the (closed) symplectic two-forms

$$
\kappa^{\alpha}=\frac{1}{2} K_{i j}^{\alpha}(\boldsymbol{z}) \mathrm{d} z^{i} \wedge \mathrm{d} z^{j},
$$

and obtain the structural conservation law (Bridges, 1997) given by

$$
\kappa_{, \alpha}^{\alpha}=0 .
$$

Hydon (2005) showed that the Poincaré Lemma leads to a one-form quasi-conservation law

$$
\left(L_{j}^{\alpha} d z^{j}\right)_{, \alpha}=\mathrm{d}\left(L_{j}^{\alpha} z_{, \alpha}^{j}-H(\boldsymbol{z})\right)=\mathrm{d} L,
$$

whose exterior derivative is (2.5).

Every one-parameter Lie group of point symmetries of the multisymplectic system (2.2) is generated by a differential operator of the form

$$
X=Q^{i}(\boldsymbol{q}, \boldsymbol{z}) \frac{\partial}{\partial z^{i}}+\left(Q^{i}(\boldsymbol{q}, \boldsymbol{z})\right)_{, \alpha} \frac{\partial}{\partial z_{, \alpha}^{i}} .
$$

Noether's Theorem implies that if $X$ generates variational symmetries, that is, if $X L=B_{, \alpha}^{\alpha}$ for some functions $B^{\alpha}$, then the interior product of $X$ with the one-form quasi-conservation law yields the conservation law

$$
\left(L_{j}^{\alpha} Q^{j}-B^{\alpha}\right)_{, \alpha}=0 .
$$

This is the multisymplectic form of Noether's theorem.

Every multisymplectic system (2.1) is invariant under translations in the independent variables $\boldsymbol{q}$. For each of these symmetries, Noether's theorem yields a conservation law

$$
\left(L_{j}^{\alpha} z_{, \beta}^{j}-L \delta_{\beta}^{\alpha}\right)_{, \alpha}=0 .
$$

Such conservation laws can equally well be obtained by pulling back the quasiconservation law (2.6) to the base space of independent variables. Commonly, the independent variables are spatial position $\boldsymbol{x}$ and time $t$. Pulling back (2.6) to these base coordinates yields the energy conservation law from the $\mathrm{d} t$ component, and the momentum conservation law from the remaining components. We shall see the form of these conservation laws for fluid dynamics in later sections. 


\section{The inverse map and Clebsch representation}

\section{(a) Lagrangian fluid dynamics and the inverse map}

Lagrangian fluid dynamics provides evolution equations for particles moving with a fluid flow. This is typically done by writing down a flow map $\Phi$ from some reference configuration to the fluid domain $\Omega$ at each instance in time. As the fluid particles cannot cavitate, superimpose or jump, this map must be a diffeomorphism.

For an $n$-dimensional fluid flow, the flow map $\Phi: \mathbb{R}^{n} \times \mathbb{R} \mapsto \mathbb{R}^{n}$ given by $\boldsymbol{x}=\Phi(\boldsymbol{l}, t)$ specifies the spatial position at time $t$ of the fluid particle that has label $\boldsymbol{l}$. The inverse map $\Phi^{-1}$ gives the label of the particle that occupies position $\boldsymbol{x}$ at time $t$ as the function $\boldsymbol{l}=\Phi^{-1}(\boldsymbol{x}, t)$. The Eulerian velocity field $\boldsymbol{u}(\boldsymbol{x}, t)$ gives the velocity of the fluid particle that occupies position $\boldsymbol{x}$ at time $t$ as follows:

$$
\dot{\boldsymbol{x}}(\boldsymbol{l}, t)=\boldsymbol{u}(\boldsymbol{x}(\boldsymbol{l}, t), t)
$$

Each label component $l_{k}(\boldsymbol{x}, t)$ satisfies the advection law

$$
l_{k, t}+u_{i} l_{k, i}=0 .
$$

Here,$t$ and ${ }_{, i}$ denote differentiation with respect to $t$ and $x_{i}$ respectively. We use Cartesian coordinates and the Euclidean inner product $\dagger$, so we shall not generally distinguish between 'up' and 'down' indices; summation from 1 to $n$ is implied whenever an index is repeated.

\section{(b) Clebsch representation using the inverse map}

A canonical variational principle for fluid dynamics may be formulated by following the standard Clebsch procedure using the inverse map (Seliger \& Whitham (1968), Holm \& Kupershmidt, 1983). The Clebsch procedure begins with a functional $\ell[\boldsymbol{u}]$ of the Eulerian fluid velocity $\boldsymbol{u}$, which is known as the reduced Lagrangian in the context of Euler-Poincaré reduction (Holm et al., 1998). One then enforces stationarity of the action $S=\int \ell[\boldsymbol{u}] \mathrm{d} t$ under the constraint that equation (3.1) is satisfied by using a vector of $n$ Lagrange multipliers, which is denoted as $\boldsymbol{\pi}$. These Lagrange multipliers are the conjugate momenta to $\boldsymbol{l}$ in the course of the Legendre transformation to the Hamiltonian formulation. One may choose $\ell[\boldsymbol{u}]$ to be solely the kinetic energy, which depends only on $\boldsymbol{u}$. More generally, $\ell$ will also depend on thermodynamic Eulerian variables such as density, whose evolution may also be accommodated by introducing constraints. These constraints are often called the "Lin constraints" (Serrin, 1959). This idea was also used in reformulating London's variational principle for superfluids (Lin, 1963).

Definition 3.1 (Clebsch variational principle using inverse map). The Clebsch variational principle using the inverse map is

$$
\delta \int_{t_{0}}^{t_{1}} \ell[\boldsymbol{u}]+\int_{\Omega} \boldsymbol{\pi} \cdot\left(\boldsymbol{l}_{, t}+\boldsymbol{u} \cdot \nabla \boldsymbol{l}\right) \mathrm{d} V(\boldsymbol{x}) \mathrm{d} t=0
$$

where $\boldsymbol{\pi}(\boldsymbol{x}, t)$ are Lagrange multipliers which enforce the constraint that particle labels $\boldsymbol{l}(\boldsymbol{x}, t)$ are advected by the flow. In bounded domains the boundary conditions

$\dagger$ This is only done for clarity and the equations are easily extended to the case when the domain $\Omega$ is a curved manifold. 
are $\boldsymbol{u} \cdot \boldsymbol{n}=0$ together with any boundary conditions associated with the definition of the functional $l[\boldsymbol{u}]$.

Taking the indicated variations leads to the following equations:

$$
\begin{aligned}
\delta \boldsymbol{u}: & \frac{\delta \ell}{\delta \boldsymbol{u}}+(\nabla \boldsymbol{l})^{T} \cdot \boldsymbol{\pi}=0, \\
\delta \boldsymbol{\pi}: & \boldsymbol{l}_{, t}+(\boldsymbol{u} \cdot \nabla) \boldsymbol{l}=0, \\
\delta \boldsymbol{l}: & \boldsymbol{\pi}_{, t}+\nabla \cdot(\boldsymbol{u} \boldsymbol{\pi})=0,
\end{aligned}
$$

where

$$
\left((\nabla \boldsymbol{l})^{T} \cdot \boldsymbol{\pi}\right)_{i}:=\pi_{k} l_{k, i}, \quad(\nabla \cdot(\boldsymbol{u} \boldsymbol{\pi}))_{k}:=\left(u_{j} \pi_{k}\right)_{, j},
$$

and the variational derivative $\delta \ell / \delta \boldsymbol{u}$ is defined by

$$
\ell\left[\boldsymbol{u}+\epsilon \boldsymbol{u}^{\prime}\right]=\ell[\boldsymbol{u}]+\epsilon \int_{\Omega} \frac{\delta \ell}{\delta \boldsymbol{u}} \cdot \boldsymbol{u}^{\prime} \mathrm{d} V(\boldsymbol{x})+\mathcal{O}\left(\epsilon^{2}\right) .
$$

Remark 3.2 (Clebsch representation). In the language of fluid mechanics, the expression (3.2) for the spatial momentum $\boldsymbol{m}=\delta \ell / \delta \boldsymbol{u}$ in terms of canonically conjugate variables $(\boldsymbol{l}, \boldsymbol{\pi})$ is an example of a "Clebsch representation," which expresses the solution of the EPDiff equations (see below) in terms of canonical variables that evolve by standard canonical Hamilton equations. This has been known in the case of fluid mechanics for more than 100 years. For modern discussions of the Clebsch representation for ideal fluids, see, for example, Holm $\&$ Kupershmidt (1983) and Marsden $\&$ Weinstein (1983). In the language of geometric mechanics, the Clebsch representation is a momentum map.

Remark 3.3 (Vorticity structure). In general, one may obtain equivalent equations by enforcing the advection of any physical quantity, such as circulation density (Nazarenko, 1997), for example. However, the number of constraint equations must equal the number of spatial dimensions in order to support all possible momentum distributions (and hence vorticity distributions). For example, if one only enforced the advection of one component of the fluid labels then the momentum would take the form $\pi_{1} \nabla l_{1}$ which does not cover all possible initial conditions. For more details see Holm \& Kupershmidt (1983).

Remark 3.4 (Local and global existence). The standard Clebsch representation of fluid mechanics (which only has one scalar constraint) is not globally-defined in certain circumstances, for instance if the helicity is non-zero. Indeed, it is not locally defined at points where the vorticity vanishes (see Graham \& Henvey (2000)). However, by using sufficiently many constraints, these problems can be overcome. This is the approach that we take.

(c) Particle relabelling

As the physics of fluids should be independent of the labelling of particles, one may relabel the particles (by a diffeomorphism of the flow domain) without changing the dynamics. This is called the particle relabelling symmetry; Noether's theorem applied to this symmetry leads to the Kelvin circulation theorem. See Holm et al. (1998) for a modern description. 


\section{(d) Clebsch momentum map}

Definition 3.5. A momentum map is a map $\mathbf{J}: T^{*} Q \rightarrow \mathfrak{g}^{*}$ from the cotangent bundle $T^{*} Q$ of the configuration manifold $Q$ to the dual $\mathfrak{g}^{*}$ of the Lie algebra $\mathfrak{g}$ of a Lie group $G$ that acts on $Q$. The momentum map is defined by the formula,

$$
\mathbf{J}\left(\nu_{q}\right) \cdot \xi=\left\langle\nu_{q}, \xi_{Q}(q)\right\rangle,
$$

where $\nu_{q} \in T_{q}^{*} Q$ and $\xi \in \mathfrak{g}$. In this formula $\xi_{Q}$ is the infinitesimal generator of the action of $G$ on $Q$ associated with the Lie algebra element $\xi$, and $\left\langle\nu_{q}, \xi_{Q}(q)\right\rangle$ is the natural pairing of an element of $T_{q}^{*} Q$ with an element of $T_{q} Q$.

For the case of equations (3.2-3.4), elements of $Q$ are given the labels $\boldsymbol{l}$ whilst elements of $T^{*} Q$ are given by conjugate pairs $(\boldsymbol{l}, \boldsymbol{\pi})$ of labels with their conjugate momenta.

Theorem 3.6. The Clebsch relation (3.2) defines a momentum map for the right action $\operatorname{Diff}(\Omega)$ of the diffeomorphisms of the domain $\Omega$ on the back-to-labels map $\boldsymbol{l} . \dagger$

Proof. The spatial momentum in equation (3.2) may be rewritten as a map $\boldsymbol{J}_{\Omega}$ : $T^{*} Q \mapsto \mathfrak{X}^{*}(\Omega)$ from the cotangent bundle $T^{*} Q$ to the dual $\mathfrak{X}^{*}(\Omega)$ of the vector fields $\mathfrak{X}(\Omega)$ given by $\boldsymbol{J}_{\Omega}: \boldsymbol{m} \cdot \mathrm{d} \boldsymbol{x}=-\left((\nabla \boldsymbol{l})^{T} \cdot \boldsymbol{\pi}\right) \cdot \mathrm{d} \boldsymbol{x}=-\boldsymbol{\pi} \cdot \mathrm{d} \boldsymbol{l}=:-\pi_{k} \mathrm{~d} l_{k}$. That is, $\boldsymbol{J}_{\Omega}$ maps the space of labels and their conjugate momenta $(\boldsymbol{l}, \boldsymbol{\pi}) \in T^{*} Q$ to the space of one-form densities $\boldsymbol{m} \in \mathfrak{X}^{*}(\Omega)$ on $\Omega$. The map may be associated with the right action $\boldsymbol{l} \cdot \eta$ of smooth invertible maps (diffeomorphisms) $\eta$ of the back-tolabels maps $\boldsymbol{l}$ by composition of functions, $\operatorname{Diff}(\Omega): \boldsymbol{l} \cdot \eta=\boldsymbol{l} \circ \eta$. The infinitesimal generator of this right action is obtained from its definition, as

$$
X_{\Omega}(\boldsymbol{l}):=\left.\frac{d}{d s}\right|_{s=0}(\boldsymbol{l} \circ \eta(s))=T \boldsymbol{l} \circ X,
$$

in which the vector field $X \in \mathfrak{X}(\Omega)$ is tangent to the curve of diffeomorphisms $\eta_{s}$ at the identity $s=0$. Thus, pairing the map $\boldsymbol{J}_{\Omega}$ with the vector field $X \in \mathfrak{X}(\Omega)$ yields

$$
\begin{aligned}
\left\langle\boldsymbol{J}_{\Omega}(\boldsymbol{l}, \boldsymbol{\pi}), X\right\rangle & =-\langle\boldsymbol{\pi} \cdot \mathrm{d} \boldsymbol{l}, X\rangle=-\int_{S} \pi_{k} l_{k, j} X_{j}(\boldsymbol{x}) \mathrm{d} V(\boldsymbol{x}) \\
& =-\langle(\boldsymbol{l}, \boldsymbol{\pi}), T \boldsymbol{l} \cdot X\rangle=-\left\langle(\boldsymbol{l}, \boldsymbol{\pi}), X_{\Omega}(\boldsymbol{l})\right\rangle
\end{aligned}
$$

where $\langle\cdot, \cdot\rangle: T_{l}^{*} Q \times T_{l} Q \mapsto \mathbb{R}$ is the $L^{2}$ pairing of an element of $T_{l}^{*} Q$ (a one-form density) with an element of $T_{l} Q$ (a vector field). This is the defining relation for (3.6) to be a momentum map.

Remark 3.7. Being the cotangent lift of the action of $\operatorname{Diff}(\Omega)$, the momentum map $\mathbf{J}_{\Omega}$ is equivariant and Poisson. That is, substituting the canonical Poisson bracket into the momentum map definition yields the Lie-Poisson bracket on the space of $\boldsymbol{m}$ 's. See, for example, Holm \& Kupershmidt (1983) and Marsden \& Weinstein (1983) for more explanation, discussion and applications. The momentum

$\dagger$ As we discuss later, this right action contrasts with fluid particle relabelling, which arises by the left action of the diffeomorphisms on the inverse map. 
map property of the Clebsch representation guarantees that the canonically conjugate variables $(\boldsymbol{l}, \boldsymbol{\pi})$ may be eliminated in favour of the spatial momentum $\boldsymbol{m}$. Before its momentum map property was understood, the use of the Clebsch representation to eliminate the canonical variables in favour of Eulerian fluid variables was a tantalising mystery (Seliger 85 Whitham, 1968).

Note that the right action of $\operatorname{Diff}(\Omega)$ on the inverse map is not a symmetry. In fact, as we shall see, the right action of $\operatorname{Diff}(\Omega)$ on the inverse map generates the fluid motion itself.

\section{(e) Advected quantities}

To construct more general fluid equations we shall include advected quantities $a$ whose flow-rules are defined by

$$
a_{, t}+\mathcal{L}_{\boldsymbol{u}} a=0
$$

where $\mathcal{L}_{\boldsymbol{u}}$ is the Lie derivative. Such advected variables typically arise in the potential energy or the thermodynamic internal energy of an ideal fluid. For example, advected scalars $s$ (salinity, for example) satisfy

$$
\frac{\partial}{\partial t} s+\mathcal{L}_{\boldsymbol{u}} s=0, \quad \text { i.e. } \quad s_{, t}+(\boldsymbol{u} \cdot \nabla) s=0,
$$

and advected densities $\rho \mathrm{d} V$ satisfy

$$
\frac{\partial}{\partial t}(\rho \mathrm{d} V)+\mathcal{L}_{\boldsymbol{u}}(\rho \mathrm{d} V)=0, \quad \text { i.e. } \quad \rho_{, t}+\nabla \cdot(\rho \boldsymbol{u})=0 .
$$

A more extensive list of different types of advected quantity is given in Holm et al. (1998).

We write the reduced Lagrangian $\ell$ as a functional of the Eulerian fluid variables $\boldsymbol{u}$ and $a$, and add further constraints to the action $S$ to account for their advection relations,

$$
S=\int \ell[\boldsymbol{u}, a] \mathrm{d} t+\int \mathrm{d} t \int_{\Omega} \boldsymbol{\pi} \cdot\left(\boldsymbol{l}_{, t}+(\boldsymbol{u} \cdot \nabla) \boldsymbol{l}\right)+\phi\left(a_{, t}+\mathcal{L}_{\boldsymbol{u}} a\right) \mathrm{d} V(\boldsymbol{x}) .
$$

The Euler-Lagrange equations, which follow from the stationarity condition $\delta S=0$, are

$$
\begin{aligned}
\delta \boldsymbol{u}: & \frac{\delta \ell}{\delta \boldsymbol{u}}+(\nabla \boldsymbol{l})^{T} \cdot \boldsymbol{\pi}+\phi \diamond a=0, \\
\delta \boldsymbol{\pi}: & \boldsymbol{l}_{, t}+(\boldsymbol{u} \cdot \nabla) \boldsymbol{l}=0, \\
\delta \boldsymbol{l}: & -\boldsymbol{\pi}_{, t}-\nabla \cdot(\boldsymbol{u} \boldsymbol{\pi})=0, \\
\delta \phi: & a_{, t}+\mathcal{L}_{\boldsymbol{u}} a=0, \\
\delta a: & -\phi_{, t}-\mathcal{L}_{\boldsymbol{u}} \phi+\frac{\delta \ell}{\delta a}=0,
\end{aligned}
$$

where the diamond operator $(\diamond)$ is defined as the dual of the Lie derivative operation $\mathcal{L}_{\boldsymbol{u}}$ with respect to the $L^{2}$ pairing. Explicitly, under integration by parts,

$$
\int_{\Omega}(\phi \diamond a) \cdot \boldsymbol{u} \mathrm{d} V(\boldsymbol{x})=-\int_{\Omega}\left(\phi \mathcal{L}_{\boldsymbol{u}} a\right) \mathrm{d} V(\boldsymbol{x}) .
$$


Remark 3.8. The map to the spatial momentum in equation (3.12)

$$
\frac{\delta \ell}{\delta \boldsymbol{u}}=: \boldsymbol{m}=-\pi_{A} \nabla l^{A}-\phi \diamond a
$$

is again a momentum map, this time for the semidirect-product action of the diffeomorphisms on $\Omega \times V^{*}$. Again the momentum map property allows the canonical variables to be eliminated in favour of the Eulerian quantities. As a result, eliminating the variables $\boldsymbol{l}, \boldsymbol{\pi}$ and $\phi$ leads to the Euler-Poincaré equation with advected quantities a.

\section{(f) Elimination theorem}

Eliminating the canonically conjugate variables $(\boldsymbol{l}, \boldsymbol{\pi})$ and $\phi$ produces an equation of motion for $\boldsymbol{m}=\delta \ell / \delta \boldsymbol{u}$, which is constructed in the proof of the following theorem:

Theorem 3.9 (Elimination theorem with advected quantities). The labels $(\boldsymbol{l})$, their conjugate momenta $(\boldsymbol{\pi})$ and the conjugate momentum $(\phi)$ to the advected quantities (a) may be eliminated from equations (3.12-3.14) to obtain the weak form of the Euler-Poincaré equation with advected quantities:

$$
\frac{\partial}{\partial t} \frac{\delta \ell}{\delta \boldsymbol{u}}+\operatorname{ad}_{\boldsymbol{u}}^{*} \frac{\delta \ell}{\delta \boldsymbol{u}}=a \diamond \frac{\delta \ell}{\delta a}, \quad a_{, t}+\mathcal{L}_{\boldsymbol{u}} a=0
$$

Proof. Take the time-derivative of the inner product of $\delta \ell / \delta \boldsymbol{u}$ with a test function $\boldsymbol{w}$ :

$$
\begin{aligned}
\frac{d}{d t}\left\langle\frac{\delta \ell}{\delta \boldsymbol{u}}, \boldsymbol{w}\right\rangle= & \frac{d}{d t}\left\langle-(\nabla \boldsymbol{l})^{T} \cdot \boldsymbol{\pi}-\phi \diamond a, \boldsymbol{w}\right\rangle=\frac{d}{d t}\langle-\boldsymbol{\pi},(\boldsymbol{w} \cdot \nabla) \boldsymbol{l}\rangle+\frac{d}{d t}\left\langle\phi, \mathcal{L}_{\boldsymbol{w}} a\right\rangle \\
& +\left\langle\phi, \mathcal{L}_{\boldsymbol{u}} \mathcal{L}_{\boldsymbol{w}} a-\mathcal{L}_{\boldsymbol{w}} \mathcal{L}_{\boldsymbol{u}} a\right\rangle \\
= & \left\langle\boldsymbol{\pi},-\left(\operatorname{ad}_{\boldsymbol{u}} \boldsymbol{w} \cdot \nabla\right) \boldsymbol{l}\right\rangle+\left\langle\phi, \mathcal{L}_{\mathrm{ad}_{\boldsymbol{u}} \boldsymbol{w}} a\right\rangle+\left\langle\frac{\delta \ell}{\delta a} \diamond a, \boldsymbol{w}\right\rangle, \\
= & \left\langle-(\nabla \boldsymbol{l})^{T} \cdot \boldsymbol{\pi}-\phi \diamond a, \operatorname{ad}_{\boldsymbol{u}} \boldsymbol{w}\right\rangle+\left\langle\frac{\delta \ell}{\delta a} \diamond a, \boldsymbol{w}\right\rangle, \\
= & \left\langle\frac{\delta \ell}{\delta \boldsymbol{u}}, \operatorname{ad}_{\boldsymbol{u}} \boldsymbol{w}\right\rangle+\left\langle\frac{\delta \ell}{\delta a} \diamond a, \boldsymbol{w}\right\rangle=\left\langle-\operatorname{ad}_{\boldsymbol{u}}^{*} \frac{\delta \ell}{\delta \boldsymbol{u}}+\frac{\delta \ell}{\delta a} \diamond a, \boldsymbol{w}\right\rangle .
\end{aligned}
$$

Remark 3.10. These Euler-Poincaré equations with advected quantities cover all conservative fluid equations which describe the advection of material. For a large collection of examples, see Holm et al. (1998). 
(g) Example: EPDiff( $\left(H^{1}\right)$

To give a concrete example, consider EPDiff with $\ell[\boldsymbol{u}]$ being the $H_{\lambda}^{1}$-norm for $\boldsymbol{u}$. This is the $n$-dimensional Camassa-Holm (CH) equation (Camassa \& Holm, 1993; Holm et al., 1998; Holm \& Marsden, 2004), which has applications in computational anatomy (Holm et al., 2004; Miller et al., 2002). This system has the reduced Lagrangian

$\ell[\boldsymbol{u}]=\int_{\Omega} \frac{1}{2}\left(|\boldsymbol{u}|^{2}+\lambda^{2}|\nabla \boldsymbol{u}|^{2}\right) \mathrm{d} V(\boldsymbol{x})=\int_{\Omega} \frac{1}{2}\left(u_{i} u_{i}+\lambda^{2} u_{i, j} u_{i, j}\right) \mathrm{d} V(\boldsymbol{x}) \equiv \frac{1}{2}\|\boldsymbol{u}\|_{H_{\lambda}^{1}}^{2}$,

which defines the $H_{\lambda}^{1}$-norm for $\lambda>0$. The EPDiff equation amounts to

$$
\frac{\partial \boldsymbol{m}}{\partial t}+(\boldsymbol{u} \cdot \nabla) \boldsymbol{m}+(\nabla \boldsymbol{u})^{T} \cdot \boldsymbol{m}+\boldsymbol{m} \nabla \cdot \boldsymbol{u}=0, \quad \boldsymbol{m}=\left(1-\lambda^{2} \nabla^{2}\right) \boldsymbol{u} .
$$

When $n=1$, these reduce to the Camassa-Holm $(\mathrm{CH})$ equation,

$$
m_{, t}+u m_{, x}+2 m u_{, x}=0, \quad m=u-\lambda^{2} u_{, x x} .
$$

\section{(h) Example: Incompressible Euler equations}

As an example, consider the reduced Lagrangian for the incompressible Euler equations

$$
\ell[\boldsymbol{u}, \rho, p]=\int_{\Omega} \frac{\rho|\boldsymbol{u}|^{2}}{2}+p(1-\rho) \mathrm{d} V(\boldsymbol{x}) .
$$

Here $\rho(\boldsymbol{x}, t)$ is the ratio of the local fluid density to the average density over $\Omega$; this is governed by the continuity equation (3.10). The pressure $p$ is a Lagrange multiplier that fixes the incompressibility constraint $\rho=1$. The variational derivatives in this case are

$$
\frac{\delta \ell}{\delta \boldsymbol{u}}=\rho \boldsymbol{u}, \quad \frac{\delta \ell}{\delta \rho}=\frac{|\boldsymbol{u}|^{2}}{2}-p, \quad \frac{\delta \ell}{\delta p}=1-\rho .
$$

Consequently, the Euler-Poincaré equations become

$$
\begin{aligned}
(\rho \boldsymbol{u})_{, t}+(\boldsymbol{u} \cdot \nabla)(\rho \boldsymbol{u})+\rho \boldsymbol{u}(\nabla \cdot \boldsymbol{u})+\rho(\nabla \boldsymbol{u})^{T} \cdot \boldsymbol{u} & =\rho \nabla\left(\frac{|\boldsymbol{u}|^{2}}{2}-p\right), \\
\rho_{, t}+\nabla \cdot(\rho \boldsymbol{u}) & =0, \\
\rho & =1,
\end{aligned}
$$

and rearrangement gives the Euler fluid equations,

$$
\boldsymbol{u}_{, t}+(\boldsymbol{u} \cdot \nabla) \boldsymbol{u}=-\nabla p, \quad \nabla \cdot \boldsymbol{u}=0 .
$$

\section{Inverse map multisymplectic formulation for the Euler-Poincaré equations of fluid dynamics}

As we now have a canonical variational principle for fluid dynamics via the inverse map, one may obtain its multisymplectic formulation by extending the phase space so that the Lagrangian is affine in the space and time derivatives. In this section we show how to do this for $\operatorname{EPDiff}\left(H^{1}\right)$ as discussed in the previous section, and then show how to generalise this to equations with advected quantities, using the incompressible Euler equations as an example. 
(a) Affine Lagrangian for EPDiff( $\left(H^{1}\right)$

After introducing the inverse map constraint, the Lagrangian becomes

$$
L=\frac{1}{2} u_{i} u_{i}+\frac{\lambda^{2}}{2} u_{i, j} u_{i, j}+\pi_{k}\left(l_{k, t}+u_{j} l_{k, j}\right) .
$$

Any high-order derivatives and nonlinear functions of first-order derivatives must now be removed from the Lagrangian to make it affine. We introduce a tensor variable

$$
W_{i j}=u_{i, j}
$$

this relationship may be enforced by using Lagrange multipliers. However, it turns out that the multipliers can be eliminated and the Lagrangian becomes

$$
L=\frac{1}{2} u_{i} u_{i}-\frac{\lambda^{2}}{2} W_{i j} W_{i j}+\lambda^{2} W_{i j} u_{i, j}+\pi_{k}\left(l_{k, t}+u_{j} l_{k, j}\right),
$$

which is now affine in the space and time derivatives of $\boldsymbol{u}, W, \boldsymbol{l}$ and $\boldsymbol{\pi}$.

\section{(b) Multisymplectic structure}

The Euler-Lagrange equations for the affine Lagrangian (4.1) are

$$
\begin{aligned}
\delta u_{i}: & u_{i}-\lambda^{2} W_{i j, j}+\pi_{k} l_{k, i}=0, \\
\delta l_{k}: & -\pi_{k, t}-\left(\pi_{k} u_{j}\right)_{, j}=0 . \\
\delta \pi_{k}: & l_{k, t}+u_{j} l_{k, j}=0, \\
\delta W_{i j}: & -\lambda^{2} W_{i j}+\lambda^{2} u_{i, j}=0 .
\end{aligned}
$$

These equations possess the following multisymplectic structure as in equation (2.2):

$$
\left(\begin{array}{cccc}
0 & \pi_{k} \partial_{i} & & -\lambda^{2} \partial_{j} \\
-\pi_{k} \partial_{i} & 0 & -\partial_{t}-u_{j} \partial_{j} & 0 \\
0 & \partial_{t}+u_{j} \partial_{j} & 0 & 0 \\
\lambda^{2} \partial_{j} & 0 & 0 & 0
\end{array}\right)\left(\begin{array}{c}
u_{i} \\
l_{k} \\
\pi_{k} \\
W_{i j}
\end{array}\right)=\nabla H
$$

where $\partial_{t}=\partial / \partial t, \partial_{i}=\partial / \partial x_{i}$, and

$$
H=-\left(\frac{1}{2} u_{i} u_{i}-\frac{\lambda^{2}}{2} W_{i j} W_{i j}\right)=-\left(\frac{1}{2}|\boldsymbol{u}|^{2}-\frac{\lambda^{2}}{2}|W|^{2}\right) .
$$

(c) One-form quasi-conservation law

For our multisymplectic formulation of $\operatorname{EPDiff}\left(H^{1}\right)$, the independent variables are

$$
q^{j}=x_{j}, \quad j=1, \ldots n, \quad q^{n+1}=t,
$$

and the dependent variables are

$$
z^{i}=u_{i}, \quad z^{n+k}=l_{k}, \quad z^{2 n+k}=\pi_{k}, \quad z^{(i+2) n+j}=W_{i j},
$$


where $i, j$ and $k$ range from 1 to $n$. Comparing (4.1) with (2.1) gives the following non-zero components $L_{j}^{\alpha}$ :

$$
L_{i}^{j}=\lambda^{2} W_{i j}, \quad L_{n+k}^{j}=\pi_{k} u_{j}, \quad L_{n+k}^{n+1}=\pi_{k}, \quad i, j, k=1, \ldots, n .
$$

Therefore the one-form quasi-conservation law amounts to

$$
\left(\pi_{k} \mathrm{~d} l_{k}\right)_{, t}+\left(\lambda^{2} W_{i j} \mathrm{~d} u_{i}+\pi_{k} u_{j} \mathrm{~d} l_{k}\right)_{, j}=\mathrm{d} L .
$$

The exterior derivative of this expression yields the structural conservation law

$$
\left(\mathrm{d} \pi_{k} \wedge \mathrm{d} l_{k}\right)_{, t}+\left(\lambda^{2} \mathrm{~d} W_{i j} \wedge \mathrm{d} u_{i}+u_{j} \mathrm{~d} \pi_{k} \wedge \mathrm{d} l_{k}+\pi_{k} \mathrm{~d} u_{j} \wedge \mathrm{d} l_{k}\right)_{, j}=0 .
$$

\section{(d) Conservation of energy}

For EPDiff $\left(H^{1}\right)$, the $\mathrm{d} t$-component of the pullback of the one-form conservation law (4.3) gives

$$
\left(\pi_{k} l_{k, t}-L\right)_{, t}+\left(\lambda^{2} W_{i j} u_{i, t}+\pi_{k} u_{j} l_{k, t}\right)_{, j}=0 .
$$

In terms of $\boldsymbol{u}$ and its derivatives, this amounts to

$$
\left(u_{i} m_{i}-\frac{1}{2} u_{i} u_{i}-\frac{\lambda^{2}}{2} u_{i, j} u_{i, j}\right)_{, t}+\left(\lambda^{2} u_{i, j} u_{i, t}+u_{i} u_{j} m_{i}\right)_{, j}=0
$$

where

$$
m_{i}=u_{i}-\lambda^{2} u_{i, k k}
$$

This is the energy conservation law for $\operatorname{EPDiff}\left(H^{1}\right)$.

\section{(e) Conservation of momentum}

Similarly, the conservation law that is associated with translations in the $x_{i^{-}}$ direction is

$$
\left(\pi_{k} l_{k, i}\right)_{, t}+\left(\lambda^{2} W_{k j} u_{k, i}+\pi_{k} u_{j} l_{k, i}-\delta_{i j} L\right)_{, j}=0,
$$

which amounts to the momentum conservation law

$$
m_{i, t}+\left(\lambda^{2} u_{k, i} u_{k, j}-u_{j} m_{i}-\delta_{i j}\left(\frac{1}{2} u_{k} u_{k}+\frac{\lambda^{2}}{2} u_{k, l} u_{k, l}\right)\right)_{, j}=0 .
$$

(f) Conservation of vorticity

Next, consider the coefficient of each $\mathrm{d} x_{r} \wedge \mathrm{d} x_{s}$ in the pull-back of the structural (two-form) conservation law (4.4). This is

$$
\begin{aligned}
\left(\pi_{k, r} l_{k, s}-\pi_{k, s} l_{k, r}\right)_{, t}+( & \lambda^{2}\left(W_{i j, r} u_{i, s}-W_{i j, s} u_{i, r}\right)+ \\
& \left.u_{j}\left(\pi_{k, r} l_{k, s}-\pi_{k, s} l_{k, r}\right)+\pi_{k}\left(u_{j, r} l_{k, s}-u_{j, s} l_{k, r}\right)\right)_{, j}=0
\end{aligned}
$$

which amounts to

$$
\left(m_{r, s}-m_{s, r}\right)_{, t}+\left(\lambda^{2}\left(u_{i, s} u_{i, j r}-u_{i, r} u_{i, j s}\right)+\left(u_{j} m_{r}\right)_{, s}-\left(u_{j} m_{s}\right)_{, r}\right)_{, j}=0 .
$$

One can regard this as a vorticity conservation law for $\operatorname{EPDiff}\left(H^{1}\right)$; it is a differential consequence of the momentum conservation law. 


\section{(g) Particle relabelling symmetry}

As we discussed in Section 3, fluid equations in general, and EPDiff in particular, are invariant under relabelling of particles. In the context of the inverse map variables, relabelling is accomplished by the action of the diffeomorphism group $\operatorname{Diff}(\Omega)$ defined by

$$
\boldsymbol{l} \mapsto \eta \circ \boldsymbol{l} \equiv \eta(\boldsymbol{l}), \quad \eta \in \operatorname{Diff}(\Omega) .
$$

The corresponding infinitesimal action of the vector fields $\mathfrak{X}(\Omega)$ is then

$$
\boldsymbol{l} \mapsto \boldsymbol{\xi} \circ \boldsymbol{l} \equiv \boldsymbol{\xi}(\boldsymbol{l}), \quad \boldsymbol{\xi} \in \mathfrak{X}(\Omega),
$$

and the cotangent lift of this action is

$$
(\boldsymbol{\pi}, \boldsymbol{l}) \mapsto\left(-(\nabla \boldsymbol{\xi}(\boldsymbol{l}))^{T} \cdot \boldsymbol{\pi}, \boldsymbol{\xi}(\boldsymbol{l})\right)
$$

To obtain the symmetry generator (2.7), we extend the above action to first derivatives as follows:

$$
\begin{aligned}
X= & \xi_{k}(\boldsymbol{l}) \frac{\partial}{\partial l_{k}}+\left(\xi_{k}(\boldsymbol{l})\right)_{, t} \frac{\partial}{\partial l_{k, t}}+\left(\xi_{k}(\boldsymbol{l})\right)_{, i} \frac{\partial}{\partial l_{k, i}} \\
& -\pi_{k} \frac{\partial \xi_{k}(\boldsymbol{l})}{\partial l_{j}} \frac{\partial}{\partial \pi_{j}}-\left(\pi_{k} \frac{\partial \xi_{k}(\boldsymbol{l})}{\partial l_{j}}\right)_{, t} \frac{\partial}{\partial \pi_{j, t}}-\left(\pi_{k} \frac{\partial \xi_{k}(\boldsymbol{l})}{\partial l_{j}}\right)_{, i} \frac{\partial}{\partial \pi_{j, i}} .
\end{aligned}
$$

The relabelling symmetries are variational, because

$$
X L=\pi_{k}\left(\xi_{k}(\boldsymbol{l})\right)_{, t}+\pi_{k} u_{i}\left(\xi_{k}(\boldsymbol{l})\right)_{, i}-\pi_{k} \frac{\partial \xi_{k}(\boldsymbol{l})}{\partial l_{j}}\left(l_{j, t}+u_{i} l_{j, i}\right)=0 .
$$

Noether's theorem then gives the conservation law

$$
\left(\pi_{k} \xi_{k}(\boldsymbol{l})\right)_{, t}+\left(\pi_{k} u_{j} \xi_{k}(\boldsymbol{l})\right)_{, j}=0 .
$$

A conservation law exists for each element $\boldsymbol{\xi}$ of $\mathfrak{X}(\Omega)$, so particle relabelling generates an infinite space of conservation laws.

\section{(h) Circulation theorem}

To see how the particle relabelling conservation laws relate to conservation of circulation, note that if $\rho$ is any density that satisfies

$$
\rho_{, t}+\left(\rho u_{j}\right)_{, j}=0,
$$

then

$$
\left(\frac{\pi_{k} \xi_{k}(\boldsymbol{l})}{\rho}\right)_{, t}+u_{j}\left(\frac{\pi_{k} \xi_{k}(\boldsymbol{l})}{\rho}\right)_{, j}=0 .
$$

If we pick a loop $C(t)$ which is advected with the flow, then

$$
\frac{d}{d t} \oint_{C(t)} \frac{\pi_{k} \xi_{k}(\boldsymbol{l})}{\rho} \mathrm{d} x=0 .
$$

Article submitted to Royal Society 
For a vector field $\boldsymbol{\xi}$ which is tangent to the loop at time 0 , and satisfies $|\boldsymbol{\xi}|=1$ on the loop, then

$$
\boldsymbol{\xi} \mathrm{d} x=(\nabla \boldsymbol{l}) \cdot \mathrm{d} \boldsymbol{x}
$$

for all times $t$, and one finds

$$
\frac{d}{d t} \oint_{C(t)} \frac{\boldsymbol{\pi} \cdot(\nabla \boldsymbol{l})}{\rho} \cdot \mathrm{d} \boldsymbol{x}=0,
$$

The momentum formula (3.2) gives

$$
\frac{d}{d t} \oint_{C(t)} \frac{\left(1-\lambda^{2} \nabla^{2}\right) \boldsymbol{u}}{\rho} \cdot \mathrm{d} \boldsymbol{x}=\frac{d}{d t} \oint_{C(t)} \frac{\boldsymbol{m}}{\rho} \cdot \mathrm{d} \boldsymbol{x}=0
$$

which is the circulation theorem for EPDiff.

\section{(i) Advected quantities}

To extend this method to more general equations with advected quantities is very simple: take the Lagrangian obtained from equation (3) and add variables to represent higher-order derivatives. For the sake of brevity we shall compute one example, the incompressible Euler equations, and briefly discuss the implications for the circulation theorem.

\section{(j) Multisymplectic form of incompressible Euler equations}

We start with the reduced Lagrangian

$$
\ell[\boldsymbol{u}, p, \rho]=\int_{\Omega} \frac{1}{2} \rho u_{i} u_{i}+p(1-\rho) \mathrm{d} V(\boldsymbol{x}),
$$

where $p$ is the pressure and $\rho$ is the relative density, and add dynamical constraints to form the Lagrangian:

$$
L=\frac{1}{2} \rho u_{i} u_{i}+p(1-\rho)+\pi_{k}\left(l_{k, t}+u_{i} l_{k, i}\right)+\phi\left(\rho_{, t}+\left(\rho u_{i}\right)_{, i}\right) .
$$

This Lagrangian is already affine in the first-order derivatives, so the Euler-Lagrange equations are automatically multisymplectic in these variables:

$$
\left(\begin{array}{cccccc}
0 & 0 & \pi_{k} \partial_{i} & 0 & -\rho \partial_{i} & 0 \\
0 & 0 & 0 & 0 & -\partial_{t}-u_{i} \partial_{i} & 0 \\
-\pi_{k} \partial_{i} & 0 & 0 & -\partial_{t}-u_{i} \partial_{i} & 0 & 0 \\
0 & 0 & \partial_{t}+u_{i} \partial_{i} & 0 & 0 & 0 \\
\rho \partial_{i} & \partial_{t}+u_{i} \partial_{i} & 0 & 0 & 0 & 0 \\
0 & 0 & 0 & 0 & 0 & 0
\end{array}\right)\left(\begin{array}{c}
u_{i} \\
\rho \\
l_{k} \\
\pi_{k} \\
\phi \\
p
\end{array}\right)=\nabla H
$$

where the quantity

$$
H=-\left(\frac{1}{2} \rho u_{i} u_{i}+p(1-\rho)\right)
$$

is negative of the Hamiltonian density. 


\section{(k) Circulation theorem for advected quantities}

The conservation law for particle-relabelling follows exactly as in Section 4, and we obtain equation (4.5) as before. The difference is that now the momentum formula (momentum map) is

$$
\boldsymbol{m}=\frac{\partial \ell}{\partial \boldsymbol{u}}=-\pi_{k} \nabla l_{k}-\phi \diamond a
$$

and so one obtains

$$
\frac{d}{d t} \oint_{C(t)} \frac{\boldsymbol{m}}{\rho} \cdot \mathrm{d} \boldsymbol{x}=\oint_{C(t)} \frac{1}{\rho} \frac{\partial \ell}{\partial a} \diamond a \cdot \mathrm{d} \boldsymbol{x} .
$$

For the incompressible Euler equations, $a$ is the relative density $\rho$, so

$$
\frac{\partial \ell}{\partial a} \diamond a=\rho \nabla \frac{\partial \ell}{\partial \rho},
$$

which leads to the circulation theorem

$$
\frac{d}{d t} \oint_{C(t)} \frac{\boldsymbol{m}}{\rho} \cdot \mathrm{d} \boldsymbol{x}=\oint_{C(t)} \nabla \frac{\partial \ell}{\partial \rho} \cdot \mathrm{d} \boldsymbol{x}=0 .
$$

Remark 4.1 ('Hidden' conservation law). Yakhot 85 Zakharov (1997) discuss a 'hidden' conservation law, which amounts to the preservation of the integral of $|\boldsymbol{\pi}|^{2}$ over label space. Similarly the label-space integral of any differentiable function $F(|\boldsymbol{\pi}|)$ over label space is preserved. This corresponds to the local conservation law

$$
(F(|\boldsymbol{\pi}|))_{, t}+\left(u_{j} F(|\boldsymbol{\pi}|)\right)_{, j}=0
$$

which is a consequence of (3.13) and the incompressibility condition.

\section{A note on multisymplectic integrators}

In this section we discuss briefly how to produce multisymplectic numerical integrators, using the inverse map formulation given in this paper. We note in particular that the multisymplectic method may have discrete forms of some of the particle-relabelling symmetries and hence we will obtain a method that has discrete conservation laws.

\section{(a) Variational integrators}

A multisymplectic integrator for a PDE is a numerical method which preserves a discrete conservation law for the two-form $\kappa$ given in equation (2.4) (Bridges \& Reich, 2001). As described in (Hydon, 2005), a discrete variational principle with a Lagrangian that is affine in first-order differences automatically leads to a set of difference equations which conserve the multisymplectic two-form. This now makes it very simple to construct multisymplectic integrators for fluid dynamics using the inverse map formulation: one simply replaces the spatial and time integrals in the action with numerical quadratures, replaces the first-order derivatives by differences, and takes variations following the standard variational integrator approach (Lew et al., 2003). Whilst the method will preserve the discrete conservation law for the two-form $\kappa$, the one-form quasi-conservation law will not be preserved in general, and hence the other conservation laws will not be exactly preserved. 


\section{(b) Discrete relabelling symmetry}

As $\boldsymbol{\pi}$ and $\boldsymbol{l}$ are still continuous in the discretised equations, the multisymplectic integrator will have discrete particle-relabelling symmetries analogous to those given in Section 4, provided that discretisation maintains the symmetry. For example, take the following centred differencing scheme for the advection constraint $\hat{L}$ of the Lagrangian L (choosing one space dimension for simplicity):

$$
\hat{L}=\pi_{i+1 / 2}^{n+1 / 2}\left(\frac{l_{i+1 / 2}^{n+1}-l_{i+1 / 2}^{n}}{\Delta t}-u_{i+1 / 2}^{n+1 / 2} \frac{l_{i+1}^{n+1 / 2}-l_{i}^{n+1 / 2}}{\Delta x}\right),
$$

where $\left(\pi_{i}^{n}, l_{i}^{n}\right)$ are the approximations to $(\pi(i \Delta x, n \Delta t), l(i \Delta x, n \Delta t))$ and where $n+$ $1 / 2$ and $i+1 / 2$ indices indicate averaging over time-levels $n$ and $n+1$, and averaging over space-levels $i$ and $i+1$, respectively. If we choose the relabelling generated by $\xi(l)=a l+b$, then the corresponding cotangent-lifted symmetry is

$$
X=(a l+b) \frac{\partial}{\partial l}-\pi a \frac{\partial}{\partial \pi}
$$

and

$$
\begin{aligned}
X \hat{L}= & \pi_{i+1 / 2}^{n+1 / 2}\left(a \frac{l_{i+1 / 2}^{n+1}-l_{i+1 / 2}^{n}}{\Delta t}-u_{i+1 / 2}^{n+1 / 2} a \frac{l_{i+1}^{n+1 / 2}-l_{i}^{n+1 / 2}}{\Delta x}\right) \\
& -a \pi_{i+1 / 2}^{n+1 / 2}\left(\frac{l_{i+1 / 2}^{n+1}-l_{i+1 / 2}^{n}}{\Delta t}-u_{i+1 / 2}^{n+1 / 2} \frac{l_{i+1}^{n+1 / 2}-l_{i}^{n+1 / 2}}{\Delta x}\right)=0 .
\end{aligned}
$$

Following the variational integrator programme described in Lew et al. (2003) the discrete form of Noether's theorem will give rise to discrete conservation laws for the multisymplectic method. It is an open question as to whether it is possible to obtain differencing schemes which are invariant under more general transformations of the label variables.

\section{Summary and Outlook}

(a) Summary

This paper describes a multisymplectic formulation of Euler-Poincaré equations (which are, in essence, fluid dynamical equations with a particle-relabelling symmetry). We have used the inverse map to obtain a canonical variational principle, following Holm \& Kupershmidt (1983). As noted in Hydon (2005), a multisymplectic formulation can be obtained by choosing variables such that the Lagrangian at most linear in the first-order derivatives, and contains no higher-order derivatives. We have shown how to construct the multisymplectic formulation for the Euler-Poincaré equations for diffeomorphisms, using the example of the $\operatorname{EPDiff}\left(H^{1}\right)$ equations, and how to extend the method to the Euler-Poincaré equations with advected quantities. These equations encompass many fluid systems, including incompressible Euler, shallow-water, Euler-alpha, Green-Naghdi, perfect complex fluids, inviscid magnetohydrodynamics, etc.

The techniques of Hydon (2005) have led to conservation laws for these systems, including the usual multisymplectic conservation laws for energy and momentum 
plus an infinite set of conservation laws which arise from the particle-relabelling symmetries of fluid dynamics. We have highlighted the connection between these latter conservation laws and Kelvin's circulation theorem, and showed that multisymplectic integrators based on this formulation may have discrete conservation laws associated with some these symmetries.

\section{(b) Outlook}

In the last section of this paper we have discussed the possibility of developing multisymplectic integrators for fluids using this framework. These ideas may aid the future development of integrators that have conservation laws for vorticity and circulation, which are desirable for numerical weather prediction and other applications. It is undoubtedly simple to construct such integrators, but the issue of accuracy with time arises whenever the flow is strongly mixing and numerical errors make the label field $\boldsymbol{l}$ very noisy. At present this restricts the applicability of these methods to problems such as nonlinear internal wave propagation which do not entangle fluid labels. One potential solution would be to try to construct an integrator where the conjugate variables $(\boldsymbol{\pi}, \boldsymbol{l})$ can be eliminated; this would only be possible if there were a finite-dimensional Lie algebra which could be used to approximate the space of vector fields.

In a different direction, we believe that multisymplectic integrators would be especially apt for applications of EPDiff to template-matching in computational anatomy (Holm et al., 2004). The matching problem is an initial-final value problem. In such problems, space and time may be treated on an equal footing, just as in the multisymplectic formulation.

\section{(c) Acknowledgements}

The work of DDH was partially supported by the Royal Society of London Wolfson Award and the US Department of Energy Office of Science ASCR. The authors would like to thank the referees for their suggested improvements to the paper.

\section{References}

Bridges, T. J. Multi-symplectic structures and wave propagation. Math. Proc. Camb. Philos. Soc., 121:147-190, 1997.

Bridges, T. J., Hydon, P. E., \& Reich, S. Multi-symplectic integrators: numerical schemes for Hamiltonian PDEs that preserve symplecticity. J. Phys. A, 38:1403-1418, 2005.

Bridges, T. J. \& Reich, S. Multi-symplectic integrators: numerical schemes for Hamiltonian PDEs that preserve symplecticity. Phys. Lett., 284A:184-193, 2001.

Camassa, R. \& Holm, D. D. An integrable shallow-water equation with peaked solitons. Physical Review Letters, 71:1661-1664, 1993.

Graham, C. R. \& Henyey, F. S. Physics of Fluids, 12:744-746, 2000.

Holm, D. D. \& Kupershmidt, B. Poisson brackets and Clebsch representations for magnetohydrodynamics, multifluid plasmas, and elasticity. Physica D, 6:347-363, 1983.

Holm, D. D. \& Marsden, J. E. Momentum maps and measure valued solutions (peakons, filaments, and sheets) of the Euler-Poincaré equations for the diffeomorphism group. 
In J. E. Marsden \& T. S. Ratiu, editors, The Breadth of Symplectic and Poisson Geometry: A Festshrift for Alan Weinstein, Birkhäuser, Boston, MA., 2004, pp. 203-235. http://arxiv.org/abs/nlin.CD/0312048.

Holm, D. D., Marsden, J. E. \& Ratiu, T. S., The Hamiltonian Structure of Continuum Mechanics in Material, Inverse Material, Spatial and Convective Representations. In Hamiltonian Structure and Lyapunov Stability for Ideal Continuum Dynamics, Univ. Montreal Press, 1986, pp. 1-124.

Holm, D. D., Marsden, J. E., \& Ratiu, T. S. The Euler-Poincaré equations and semidirect products with applications to continuum theories. Adv. in Math., 137:1-81, 1998. http://arxiv.org/abs/chao-dyn/9801015.

Holm, D. D., Rananather, J. T., Trouvé, A., \& Younes, L. Soliton dynamics in computational anatomy. NeuroImage, 23:170-178, 2004. http://arxiv.org/abs/nlin.SI/0411014.

Hydon, P. E. Multisymplectic conservation laws for differential and differential-difference equations. Proc. Roy. Soc. LOnd. A, 461:1627-1637, 2005.

Lew, A., Marsden, J. E., Ortiz, M. \& West, M. An overview of variational integrators. In L. P. Franca, T. E. Tezduyar \& A. Masud, editors, Finite Element Methods: 1970 s and Beyond. CIMNE, Barcelona, Spain, 2004, pp. 98-115.

Lin, C. C. Liquid Helium. In Proceedings of the Enrico Fermi International School of Physics, Course XXI, G. Careri, editor, Academic Press, New York, 1963, pp. 93-146.

Marsden, J., Pekarsky, S., Shkoller, S., and West, M. Variational methods, multisymplectic geometry and continuum mechanics. J. Geom. Phys. 38:253-284, 2001.

Marsden, J. E. \& Weinstein, A. Coadjoint orbits, vortices, and Clebsch variables for incompressible fluids. Physica D, 7:305-323, 1983.

Miller, M. I., Trouvé, A. \& Younes, L. On the metrics and Euler-Lagrange equations of computational anatomy. Ann. Rev. Biomed. Engrg., 4:375-405, 2002.

Nazarenko, S.V. The circulation density and its role in 3D turbulence, Physica D 102:343348, 1997.

Seliger, R. L. \& Whitham, G. B. Variational principles in continuum mechanics. Proc. Roy. Soc. Lond. A 305: 1-25, 1968.

Serrin, J. Mathematical principles of classical fluid mechanics. In S. Flügge \& C. Truesdell, editors, Handbuch der Physik VIII/I. Springer-Verlag, Berlin, 1959, pp. 125-263.

Yakhot, V. \& Zakharov, V. Hidden conservation laws in hydrodynamics, energy and dissipation rate fluctuation spectra in strong turbulence. Physica D 64 379-394, 1997. 